\title{
The Insulin Secretion of a Minced Neonatal Rat Pancreas Cultured in a Pancreatic Chamber, in Response to Various Insulin Secretagogues
}

\author{
Yoshimasa ARAKI, KUnINori YOSHIOKA, YASUNORI INOUE, \\ Yoshio NAKAMURA, NaOTo NAKAMURA, KoJI NAKANO, \\ Toshinide YOSHIDA and Motoharu KONDO \\ First Department of Internal Medicine, Kyoto Prefectural \\ University of Medicine, Kyoto, 602, Japan
}

\begin{abstract}
The minced pancreas of a neonatal rat was cultured for 35 days in a pancreatic chamber which was constructed of a plastic tube and an ultrafiltration membrane. Insulin and amylase secreted from this pancreatic chamber into the culture medium were measured. During the experiment, the concentration of glucose in the culture medium was changed between 5.5 and $16.5 \mathrm{~mm}$ at 2-3 day intervals in order to determine the insulin secretory response of the pancreatic tissue. Insulin secretion was markedly increased in response to $16.5 \mathrm{~mm}$ glucose. The ratio of insulin secretion to amylase secretion in the culture medium increased with the advance of culture days although secretions of both insulin and amylase decreased individually. On the 7 th culture day, short term incubations were performed to test with various insulin secretagogues; obvious insulin release into the incubation medium was observed. These results show that the pancreatic chamber also in vitro secretes insulin rapidly and significantly in response to various stimuli; that by longer culture of a neonatal rat pancreas in this device, insulin secretory cells without exocrine tissue would be obtained without using digestive enzymes; that application of a pancreatic chamber for a pancreatic transplantation may be feasible.
\end{abstract}

It has been reported from our laboratory (Araki et al., 1980) that ten minced neonatal rat pancreases in two pancreatic chambers each constructed of a plastic tube and an ultrafiltration membrane sufficiently ameliorated diabetes in a totally pancreatectomized young dog, and that these pancreatic chambers could prevent the rejection reaction for three weeks with some secretions of insulin in response to glucose loading. However, it was still unknown if this device could release insulin sufficiently in response to various stimuli in vitro, or if it was possible to obtain pancreatic endocrine cells without exocrine tissue in the culture system in which both pancreatic

Received July 17, 1980. endocrine and exocrine tissues were placed together. This experiment is designed to clarify these questions.

\section{Materials and Methods}

\section{Culture device}

The culture device consisted of a tissue culture dish ( $35 \mathrm{~mm}$, Corning, USA) and a pancreatic chamber whcih was constructed from the barrel of a $20-$ $\mathrm{m} l$ plastic syringe cut to a length of $0.5 \mathrm{~cm}$ with a semipermeable membrane (XM 100A ${ }^{\circledR}$, Amicon, USA) fixed over one end. Pancreatic tissue was transferred into this barrel, which was then placed inside the tissue culture dish, the cover of which was attached to the other end of the barrel with alpha-cyanoacrylate monomer (Aron alpha $A{ }^{\circledR}$, Sankyo Co., Japan) (Fig. 1). The materials for this device were sterilized before use. 


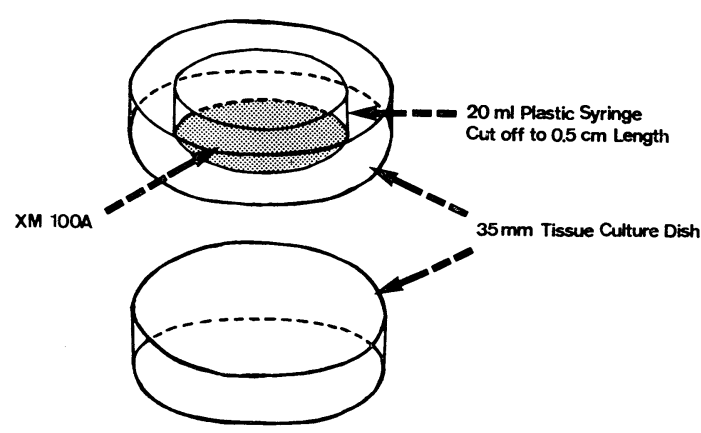

Fig. 1. Diagram shows the construction of the culture device using Pancreatic Chamber.

\section{Tissue culture}

Twenty two pancreases of five-day-old inbred Wister neonatal rats were used. Each pancreas was removed, and each was trimmed in Hank's solution, minced and put into a pancreatic chamber containing $0.5 \mathrm{ml}$ of the culture medium consisting of Medium 199 to which was added 10\% fetal bovine serum (Microbiological associates, USA), $8.4 \mathrm{mg} / 100$ $\mathrm{m} l$ of aprotinin (Trasylol@, Bayer, Germany) and $60 \mu \mathrm{g} / \mathrm{m} l$ of Tobramycin (Tobracin ${ }^{\circledR}$, Eli Lilly \& Co., LTD., USA). $2.5 \mathrm{ml}$ of the above medium was put into each culture device, and the insulin secretory response of the pancreatic tissue was observed after changing the concentration of glucose in the culture medium from 5.5 to $16.5 \mathrm{~mm}$ at $2-3$ day intervals. The device was subsequently incubated at $37^{\circ} \mathrm{C}$ in a humidified atmosphere with $5 \% \mathrm{CO}_{2}$ in air.

\section{Insulin release}

On the 7th culture day, short term incubations of the pancreatic tissue in the device were performed. Before incubation and at each interval of incubation, the device was rinsed with Krebs-Ringer bicarbonate solution (KRBS). Glucose was absent in KRBS which was used in this experiment. After preincubation with KRBS for one hour, the device was incubated with both 5.5 and $16.5 \mathrm{~mm}$ glucose in KRBS for both $15 \mathrm{~min}$ and one hour. Furthermore incubation tests with both 10 and $20 \mathrm{~mm}$ arginine in KRBS for one hour, and with both $100 \mathrm{pg} / \mathrm{m} l$ and 3CO $\mathrm{pg} / \mathrm{m} l$ of glucagon in KRBS for one hour were performed the same as for the glucose loading. Thereafter the amounts of insulin secreted into the culture medium and the incubation medium from the pancreatic chamber were measured by the radioimmunoassay technique with a double antibody method (Insulin radioimmunoassay kit, Dinabot Radioisotope Lab., Japan), and the amounts of amylase secreted into the culture medium by the blue starch method
(Amylase test ${ }^{\circledR}$, Shionogi \& Co., Ltd., Japan). The statistical differences were evaluated by the Student's $t$-test.

\section{Histological study}

After 35 days culture, the membranes used for the pancreatic chamber were fixed with Bouin's solution and embedded in paraffin. Sections were stained with either hematoxylin and eosin, or aldehyde fuchsin.

\section{Results}

The amount of insulin secreted into the culture medium consistently decreased at 2-3 day intervals, but when the concentration of glucose in the culture medium was changed from 5.5 to $16.5 \mathrm{~mm}$ the secretion of insulin was significantly enhanced $1.4 \pm 0.9$ to $2.5 \pm 1.2(\mathrm{p}<0.01), 0.9$ \pm 0.5 to $1.5 \pm 0.6(\mathrm{p}<0.01), 0.9 \pm 0.4$ to 1.2 $\pm 0.3(\mathrm{p}<0.05)$ and $0.4 \pm 0.2$ to $0.7 \pm 0.3$ $\mathrm{mU} /$ chamber-day $(\mathrm{p}<0.005)$. Day by day the secretion of amylase was also remarkably reduced from $881 \pm 22 \mathrm{IU} /$ chamber-day to below the level of detectability. But the ratio of insulin:amylase secretions had a tendency to increase substantially during the culture days (Fig. 2).

$5.5 \mathrm{~mm}$ glucose substantially increased the secretion of insulin from the pancreatic chamber into the incubation medium from $16.5 \pm 4.7$ to $33.6 \pm 5.2 \mu \mathrm{U} /$ chamber $\quad(\mathrm{p}<$ 0.05 ), and $16.5 \mathrm{~mm}$ glucose more significantly enhanced the insulin release to 59.2 $\pm 7.6 \mu \mathrm{U} /$ chamber $(\mathrm{p}<0.01$; vs $5.5 \mathrm{~mm})$ (Fig. 3a), and from $12.4 \pm 4.3$ to $42.2 \pm 6.4$ $\mu \mathrm{U} /$ chamber $(\mathrm{p}<0.005)$ even in the $15 \mathrm{~min}$ incubation (Fig. 3b). $10 \mathrm{~mm}$ arginine and $100 \mathrm{pg} / \mathrm{m} l$ of glucagon significantly increased the insulin secretion from $11 \pm 3$ and $19 \pm 4.1$ to $30.1 \pm 5.2(\mathrm{p}<0.005)$ and $54.3 \pm 6.1 \mu \mathrm{U} /$ chamber $(\mathrm{p}<0.001)$ respectively. Also 20 $\mathrm{mm}$ arginine and $300 \mathrm{pg} / \mathrm{m} l$ of glucagon induced remarkable increases in insulin secretion to $67 \pm 6.9 \quad(\mathrm{p}<0.001$; vs $10 \mathrm{~mm}$ arginine) and $89.9 \pm 8.1 \mu \mathrm{U} /$ chamber $(\mathrm{p}<$ 


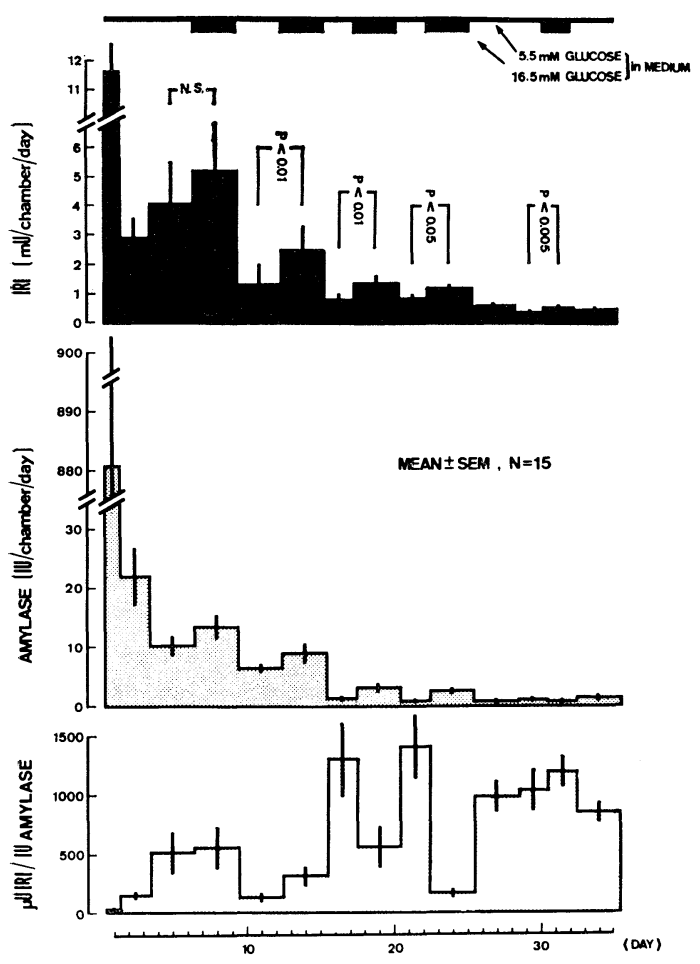

Fig. 2. The top diagram shows the pattern and rate of insulin secretion from Pancreatic Chamber in response to glucose stimulus during 35 culture days; the center diagram shows the release of amylase from Pancreatic Chamber; the bottom diagram shows the pattern and rate of secretion of insulin: amylase ratio. $(\mathrm{N}=15)$

0.005 ; vs $100 \mathrm{pg} / \mathrm{ml}$ of glucagon), respectively (Fig. 4.5).

Microscopic examination showed a few remaining pale, monolayer epithelial cells on the internal surface of the membrane, although most of the cells had degenerated. No significant fibroblastoid cells were found on the internal surface of the membrane.

\section{Discussion}

Leonard et al. $(1973 ; 1974)$ demonstrated that a neonatal rat pancreas has an extremely low exocrine enzyme content and

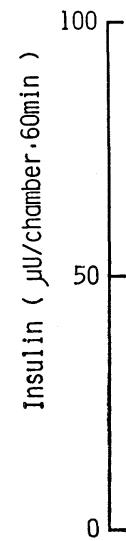

MEAN士SEM

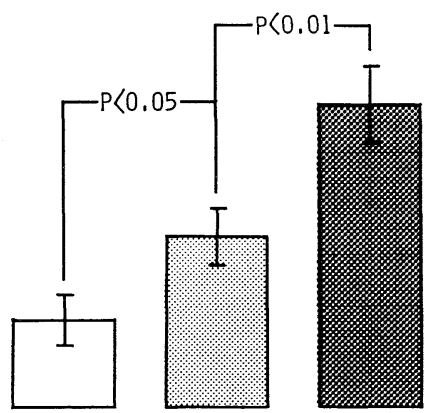

Glucose Conc.

0

5.5

16.5

Fig. 3a. Insulin secretion from Pancreatic Chamber in response to glucose loading test performed for one hour on 7 th culture day. $(\mathrm{N}=15)$
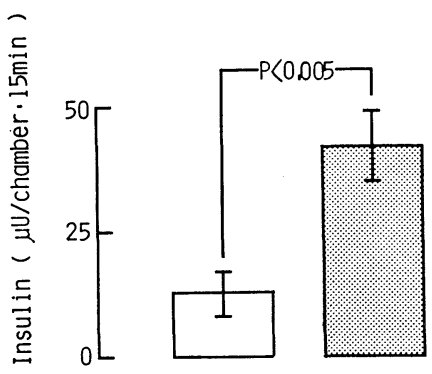

MEAN士SEM

Glucose Conc.

5.5

16.5

$(\mathrm{mM})$

Fig. 3b. Insulin secretion from Pancreatic Chamber in response to glucose loading test performed for 15 min on 7 th culture day. $(N=7)$

the dissociated neonatal rat pancreas could be transplanted without the separation of islets from exocrine elements, and that only a small quantity of islet tissue is neccessary for the amelioration of diabetes. With respect to the question of obtaining enough islets for transplantation, the collagenase digestion method (Lacy and Kostianovsky, 1967) for isolation of islets is associated 


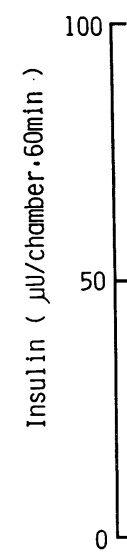

Arginine Conc. $(\mathrm{mM})$

Fig. 4. Insulin secretion from Pancreatic Chamber in response to arginine loading test performed for one hour on 7 th culture day. $(\mathrm{N}=12)$

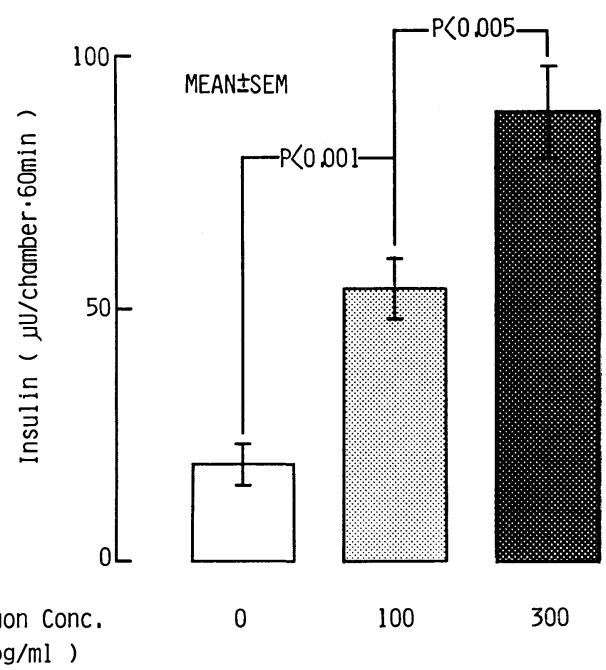

Fig. 5. Insulin secretion from Pancreatic Chamber in response to Glucagon loading test performed for one hour on 7 th culture day. $(N=10)$ with islet loss, and multiple donors are required to obtain enough islets to ameliorate diabetes in a single recipient. With respect to overcoming the rejection problem, many successful experiments have already been done (Araki et al., 1980; Gates and Lazarus, 1977; Tze et al., 1979), but observation had not been continued over a long period. This study is therefore designed to clarify whether or not it is possible to culture the pancreatic tissue in the pancreatic chamber for a long period without insulin secretory cell loss, and in addition, to estimate if this pancreatic tissue can secrete insulin rapidly and sufficiently through the artificial membrane in response to various secretagogues.

The secretion of insulin and amylase declined following the culture days but the ratio of insulin secretion to amylase secretion rapidly rose as a result of amylase loss and tissue insulin preservation as described by Matas et al. (1976) as shown in Fig. 2. However, it is still unknown why insulin secretion is preserved in comparison with amylase loss following the culture days. Exocrine cells may be autodigested rapidly in the tissue culture due to the action of intrinsic digestive enzymes. Fig. 2 also suggests that pancreatic tissue in the pancreatic chamber can secrete insulin even up to the 31 st culture day because of the fact that significant insulin secretion into the culture medium was caused by an increase in glucose concentration from 5.5 to $16.5 \mathrm{~mm}$. Therefore, by longer culture of minced neonatal rat pancreases, insulin secretory tissue without exocrine element may be obtained without a digestive method. However, using minced pancreatic tissue in the pancreatic chamber, we could not maintain the same level of insulin secretion in the advanced culture days as in the initial culture days. Taniguchi et al. (1980) suggest the possibility of keeping the level of insulin secretion at the same level as in the initial culture days by adding adenosine to the culture medium, and therefore, even in the advanced culture days, insulin could be sufficiently secreted using adenosine in 
the culture medium. Fig. 3a. 3b. 4.5. reveal that pancreatic tissue in this device can release a sufficient amount of insulin in response to various stimuli in short term incubation. Furthermore, substantial short term responses to secretagogues indicate that these pancreatic chambers have the potential to permeate both insulin and the secretagogues used in this experiment without much time-lag.

Our study shows that pancreatic tissue transplantation using a pancreatic chamber can overcome both the problem of obtaining a sufficient amount of insulin secreting tissue, and the problem of the rejection reaction.

\section{Acknowledgements}

The authors wish to thank Ms. C. P. Blue for her help in the preparation of this paper, and are grateful to Ms. I. Ohachi for her excellent technical assistance.

\section{References}

Araki, Y., Y. Inoue, K. Yoshioka, Y. Nakamura, T. Yoshida and M. Kondo (1980). Normolization of blood glucose in totally pancreatectomized dogs by use of pancreatic chambers. Endocrinol. Japon. 27, 157-161.

Leonard, R. J., A. Lazarow and O. D. Hegre (1973). Pancreatic islet transplantation in the rat. Diabetes 22, 413-428.

Leonard, R. J., A. Lazarow, R. C. McEvoy and O. D. Hegre (1947). Islet cell transplant. Kidney Int. 6, (Suppl. 1), 169-178.

Lacy, P. E. and M. Kostianovsky (1967). Method for the isolation of intact islets of Langerhans from the rat pancreas. Diabetes 16, 35-39.

Gates, R. J. and N. R. Lazarus (1977). Reversal of streptozocin-induced diabetes in rats by intraperitoneal implantation of encapsulated neonatal rabbit pacreatic tissue. Lancet II, 1257-1259.

Tze, W. J., F. C. Wong and L. M. Chen (1979). Implantable artifficial capillary unit for pancreatic islet allograft and xenograft. Diabetologia 16, 247252.

Matas, A. J., D. E. R. Surtherland and J. S. Najarian (1976). Current status of islet and pancreas transplantation in diabetes. Diabetes 25, 785-795.

Taniguchi, H., A. Tsutou and S. Baba (1980). Effectiveness of adenosine for lon-term maintenance of insulin release from cultured islets. Early Diabetes pathologenasis, diagnosis, prevention. Central Institute for Diabetes, Gerhardt Katsch, Karlsburg. $80-81$. 